\title{
Proteomic profile of visceral adipose tissues between low-fat diet-fed obesity-resistant and obesity-prone $\mathrm{C} 57 \mathrm{BL} / 6$ mice
}

\author{
WEI-DONG XIE ${ }^{1}$, HUA WANG $^{2}$, JIN-FANG ZHANG ${ }^{1,2}$, HSIANG-FU KUNG $^{2}$, YU-NAN ZHAO ${ }^{3}$ and YAOU ZHANG ${ }^{1}$ \\ ${ }^{1}$ Life Science Division, Graduate School at Shenzhen, Tsinghua University, Shenzhen; \\ ${ }^{2}$ Stanley Ho Centre for Emerging Infectious Diseases, The Chinese University of Hong Kong, Hong Kong SAR; \\ ${ }^{3}$ Laboratory of Pathological Sciences, Basic Medical College, Nanjing University of \\ Traditional Chinese Medicine, Nanjing, P.R. China
}

Received June 14, 2010; Accepted August 18, 2010

DOI: $10.3892 / \mathrm{mmr} .2010 .354$

\begin{abstract}
Certain individuals ingesting a low-fat diet are still prone to visceral obesity, and the reasons remain undetermined. This study aimed to investigate differentially expressed proteins of visceral adipose tissues between low-fat diet-fed obesity-resistant and obesity-prone C57BL/6 mice through a proteomics approach and to ascertain potential mechanisms associated with these obesity-prone animals. First, animals were fed low-fat or normal-chow diets for 6 weeks. Dietary intake and body weight were monitored during this period. After 6 weeks of the low-fat diet, obesityprone and obesity-resistant mice (NOP and NOR groups) were defined as those within the upper and lower quarters for weight gain, respectively. Proteomic analysis showed that ubiquinol-cytochrome c reductase core protein 1 (Uqcrc1) and Enolase 3, $\beta$ muscle were decreased by 8.1 - and 8.8 -fold in the visceral adipose tissues of the NOP mice compared to the NOR controls, respectively, while monoglyceride lipase (MGLL) and glucose-6-phosphate dehydrogenase (G6PDH) $\mathrm{X}$-linked were increased by 5.3 - and 4.7-fold, respectively. These results indicate that obesity-prone animals fed low-fat diets exhibited differentially expressed proteins, e.g., Uqcrc1, Enolase 3, MGLL and G6PDH involved in energy metabolism, glycolysis and fat synthesis in visceral adipose tissues, which are useful for defining molecular markers and understanding the mechanisms of the development of obesity.
\end{abstract}

\section{Introduction}

Obesity, with its devastating implications for overall health, is a serious medical problem now reaching epidemic propor-

Correspondence to: Dr Wei-Dong Xie, Life Science Division, Graduate School at Shenzhen, Tsinghua University, Shenzhen 518055, P.R. China

E-mail: xiewdong@163.com

Key words: obesity, proteomics, ubiquinol-cytochrome c reductase core protein 1, enolase 3, monoglyceride lipase, glucose-6-phosphate dehydrogenase X-linked tions worldwide (1). It is associated with an increased risk of numerous comorbidities, such as type 2 diabetes, metabolic syndrome, hypertension, cardiovascular diseases and osteoarthritis (2). However, the prevention and treatment of obesity remains a challenge, since the underlying mechanisms of pathogenesis remain undetermined.

A common idea suggests that interaction between genetic and environmental factors may contribute to the development of obesity (3). A high-fat diet is a necessary catalyst for the observed variability in body weight gain and the development of obesity (4). However, certain individuals are still rendered obese even though they are subjected to low-fat or normal-fat diets. Currently, the approach for treating obesity primarily involves caloric restriction, physical exercise and use of antiobesity drugs (1). The study of susceptibility to diet-induced obesity may be useful for identifying potential targets for preventing the development of obesity.

Many investigations have searched for candidate factors that render individuals susceptible to diet-induced obesity. Studies comparing obesity-prone and -resistant animals indicate that obesity-prone animals may favor fat storage or show a decreased fatty acid oxidation compared to obesityresistant controls (5). Different levels of neuropeptide Y and leptin may also contribute to a propensity to obesity resistance $(6,7)$. Taken together, the potential mechanisms involved in obesity resistance may include caloric intake, fat metabolism and storage. However, the exact molecular mechanisms are far from being elucidated.

Visceral adipose tissues are the main markers of obesity. Proteomics is an important method widely used in many research fields and supplies much useful information for our reference. In the present study, we investigated differentially expressed proteins of visceral adipose tissues between obesity-resistant and obesity-prone C57BL/6 mice fed low-fat diets using a proteomics method and tried to identify molecular markers and to understand the potential mechanisms of the development of obesity.

\section{Materials and methods}

Animals and diets. Three-week-old male C57BL/6 mice [SPF grade, certification no. SCXK (Guangdong) 2003-0002] 
were obtained from the Guangdong Medical Laboratory Animal Center (Guangzhou, Guangdong, China). Animals were maintained in an environmentally controlled breeding room (temperature $20 \pm 2^{\circ} \mathrm{C}$, humidity $60 \pm 5 \%, 12$-h dark/light cycle). They were fed standard laboratory chow with water ad libitum and fasted from 9:00 am to 3:00 pm prior to the experiments. Our research was conducted in accordance with the Declaration of Helsinki and/or the Guide for the Care and Use of Laboratory Animals as adopted and promulgated by the US National Institutes of Health and was approved by the Animal Ethics Committees of Tsinghua University, China. Normal-chow diets were obtained from the Guangdong Medical Laboratory Animal Center. The low-fat or normalchow diets contained $20 \%$ crude protein, $4 \%$ crude fat and $60 \%$ crude carbohydrates $(\mathrm{g} / \mathrm{g})$. Total calorie intake from the normal-chow diets was $15 \mathrm{~kJ} / \mathrm{g}$ (10\% calories in fat).

Experimental procedure. Mice 4 weeks of age had free access to water and chow diets $(n=40)$. Dietary intake and body weight was monitored once a week. Dietary and water intake, urine volume and fecal weight for each mouse were recorded in a metabolic cage (Tecniplast ${ }^{\circledR}$, Italy) for $24 \mathrm{~h}$, once a week. Total calorie intake was calculated according to the dietary calorie intake and expressed as $\mathrm{kJ} / \mathrm{g} / \mathrm{day}$. Collected feces were used for lipid assaying. After 6 weeks of low-fat diet feeding, mice ranked in the upper and lower quarters for weight gain during this feeding period were designated as obesity prone and obesity resistant (NOP and NOR, $n=10$ ), respectively. At the sixth week, animals were weighed and anesthetized by an intraperitoneal injection of pentobarbital at a dose of $35 \mathrm{mg}$ / $\mathrm{kg}$. Blood was collected from the tail vein prior to and from the orbital plexus after the 6-week treatment, respectively. Serum was isolated by centrifugation at $1,500 \mathrm{x} \mathrm{g}$ at $4^{\circ} \mathrm{C}$ for $10 \mathrm{~min}$ and stored at $-80^{\circ} \mathrm{C}$ until used for blood glucose and lipid assays. Following blood collection, anesthetized mice were sacrificed by cervical dislocation. Visceral adipose tissues (perigonadal fat, the main part of the internal white adipose tissues) were removed from the animals and immediately weighed. These samples were instantly frozen in liquid nitrogen and then stored at $-80^{\circ} \mathrm{C}$ until used for biochemical analysis.

Biochemical analysis. Blood glucose and triglycerides, total cholesterol, low-density lipoprotein cholesterol (LDL-c) and high-density lipoprotein cholesterol (HDL-c) $(n=10)$ were estimated using commercial kits (BioSino Bio-Technology and Science Inc., Beijing, China) (8-12). Fecal lipid assays were conducted according to the following protocol. Collected feces were dried at $60^{\circ} \mathrm{C}$ to a constant weight. Dried feces (100-200 mg) were combined with $0.2 \mathrm{ml}$ of PBS $(0.1 \mathrm{M}$, $\mathrm{pH} 7.4$ ) and $1 \mathrm{ml}$ of $\mathrm{CHCl}_{3}-\mathrm{CH}_{3} \mathrm{OH}(2: 1)$ in a $1.5-\mathrm{ml}$ Eppendorf tube and homogenized. The homogenates were centrifuged at $10,000 \mathrm{x} \mathrm{g}$ for $5 \mathrm{~min}$. A $0.1-\mathrm{ml}$ aliquot of the lower phase was added to another Eppendorf tube and air-dried. The dried lipids were assayed with commercial clinical diagnosis kits according to the above methods. Data were expressed as $\mu \mathrm{g} /$ mg feces.

2-Dimensional electrophoresis. Freshly prepared protein samples from the visceral adipose tissues of 3 randomly selected animals in each group were analyzed by 2-dimensional electrophoresis (2DE). Protein extraction of adipose tissues, IEF and second dimensional separation of samples, gel staining and image analysis, trypticin-gel digestion, MALDI-TOF/TOF mass spectrometric analysis and database search for protein identification were carried out according to previously described methods (13). Briefly, the cell pellets were re-suspended in lysis buffer (8 M urea, $2 \mathrm{M}$ thiourea, $2 \%$ CHAPS, 1\% NP-40, 2 mM TBP, 1X Protease Inhibitor Mix, 1X Nuclease Mix, $1 \mathrm{mM}$ PMSF and 2\% IPG buffer) and held on ice for $45 \mathrm{~min}$. The lysate was then centrifuged at $16,000 \mathrm{x} \mathrm{g}$ for $30 \mathrm{~min}$ at $4^{\circ} \mathrm{C}$, and the supernatant was saved and stored at $-80^{\circ} \mathrm{C}$ until use. IEF was carried out using an IPGphor II apparatus (Ammersham). IPG strips $(13 \mathrm{~cm}$, $\mathrm{pH} 3.0$ to 10.0 , NL) were used according to the manufacturer's instructions. Samples containing $150 \mu \mathrm{g}$ protein were diluted to $250 \mu \mathrm{l}$ in rehydration solution (8 $\mathrm{M}$ urea, 2\% CHAPS, $0.4 \%$ DTT, $0.5 \%$ IPG buffer and $0.002 \%$ bromophenol blue). The rehydration step was carried out with $13-\mathrm{cm}$ IPG strips for $10 \mathrm{~h}$ at a low voltage of $30 \mathrm{~V}$. IEF was run following a step-wise voltage increase procedure: 500 and $1,000 \mathrm{~V}$ for $1 \mathrm{~h}$ each and 8,000 V for $64 \mathrm{KVh}$. After IEF, IPG gel strips were placed in an equilibration buffer (6 M urea, 2\% SDS, 30\% glycerol, $0.002 \%$ bromophenol blue, $50 \mathrm{mM}$ Tris-HCl, $\mathrm{pH}$ 6.8) containing $1 \%$ DTT for 15 min under agitation. The IPG strips were then transferred to the equilibration solution containing $2.5 \%$ iodoacetamide and shaken for a further 15 min before applying them to $12.5 \%$ uniform polyacrylamide gel slabs $(150 \times 158 \times 1.5 \mathrm{~mm})$. Separation in the second dimension was performed in Tris-glycine buffer (25 mM Tris, $0.2 \mathrm{M}$ glycine and $0.1 \% \mathrm{SDS}$ ) at a constant current setting of $15 \mathrm{~mA} / \mathrm{gel}$ for $30 \mathrm{~min}$ and $30 \mathrm{~mA} / \mathrm{gel}$ thereafter. After 2DE, the gels were stained by a modified silver-staining method which was compatible with the following MS analysis. Spots of interest were manually excised from the 2D gels. Mass spectrometric analysis was carried out using a MALDI-TOF/TOF tandem mass spectrometer ABI 4700 proteomics analyzer (Applied Biosystems, USA). In order to perform protein identification, the MS (peptide-mass-fingerprint approach) and MS/MS (DeNovo sequencing approach) data were loaded into the GPS Explorer $^{\mathrm{TM}}$ software v3.5 (Applied Biosystems) and searched against NCBInr 5825255 sequences (released on Jan 10, 2008) by Mascot search engine v1.9.05 (Matrix Science, UK).

Statistical analysis. Data were expressed as the mean \pm SD. Statistical analysis was performed using one-way analysis of variance (ANOVA). The Newman-Keuls comparisons were used to determine the source of significant differences where appropriate. P-values of $<0.05$ were considered statistically significant.

\section{Results}

Body weight, visceral fat index, dietary intake and fecal lipid excretion. Following 6 weeks of exposure to low-fat diets, the NOP mice had a significantly increased body weight $(\mathrm{P}<0.01)$ and visceral adipose tissue $(\mathrm{P}<0.01)$ compared to the NOR controls (Fig. 1). Caloric intake plays an important role in determining the differences in body weight between individuals. After normalization of body weight, no significant 
A

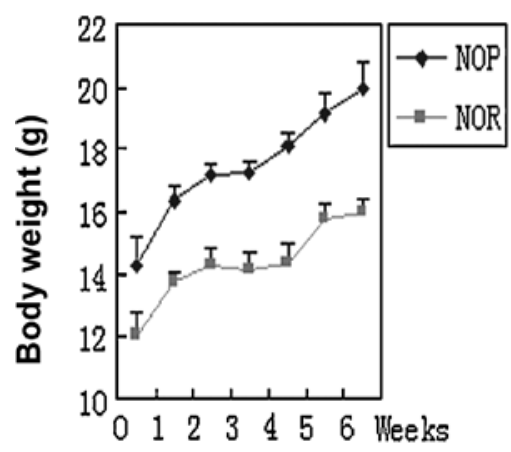

B

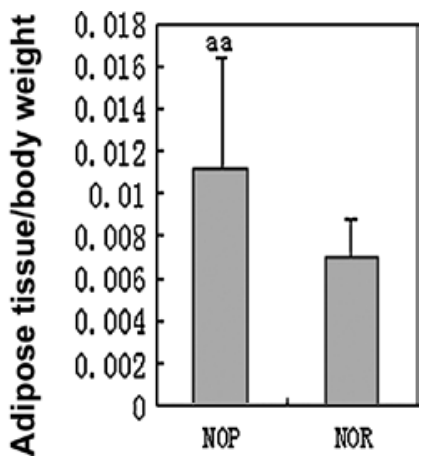

Figure 1. Changes in body weight (A) and visceral fat weight (B) in the obesity-prone and -resistant mice fed normal chow diets for 6 weeks. NOP, low-fat or normal-chow diet-fed obesity-prone mice; NOR, low-fat or normal-chow diet-fed obesity-resistant mice. Data were expressed as the mean \pm $\mathrm{SD}(\mathrm{n}=10)$. ${ }^{\text {aa }} \mathrm{P}<0.01$ vs. NOR.

difference was observed between the two groups (data not shown). Water intake, fecal and urine excretions showed similar changes to dietary caloric intake between the groups (data not shown). Total calories from fecal fat were crudely calculated (according to fecal weights and fecal triglycerides) and totalled $<1 \%$ of the total dietary caloric intake (data not shown), which suggested that caloric differences of intestinal lipid absorption were negligible between the mouse groups.

Serum biochemical parameters. No significant changes in blood glucose or lipid levels were observed between the NOP and NOR mice ( $\mathrm{P}>0.05)$ at any week (data not shown).

Differentially expressed proteins determined through 2-dimensional electrophoresis. Considering that adipose tissues serve as the main marker of obesity, we conducted $2 \mathrm{DE}$ in adipose tissues in order to find some direct clues to explain the differences between obesity-prone and -resistant animals. Proteins were regarded as differentially expressed when the magnitude of the difference was $>2$-fold and the result was reproduced twice. As a result, 16 proteins were found differentially expressed and well-identified in adipose tissues between the NOP and NOR mice (Table I). The NOP mice showed a significant change in the expression of proteins responsible for the cell skeleton, energy metabolism, oxidative stress and undefined functions compared to the NOR controls. Full images of 2DE are shown in Fig. 2. Protein spots of interest (those directly contributing to energy metabolism) are marked and enlarged in Fig. 3. Ubiquinol-cytochrome $\mathrm{c}$ reductase core protein 1 (Uqcrc1) expression (related to mito-
A

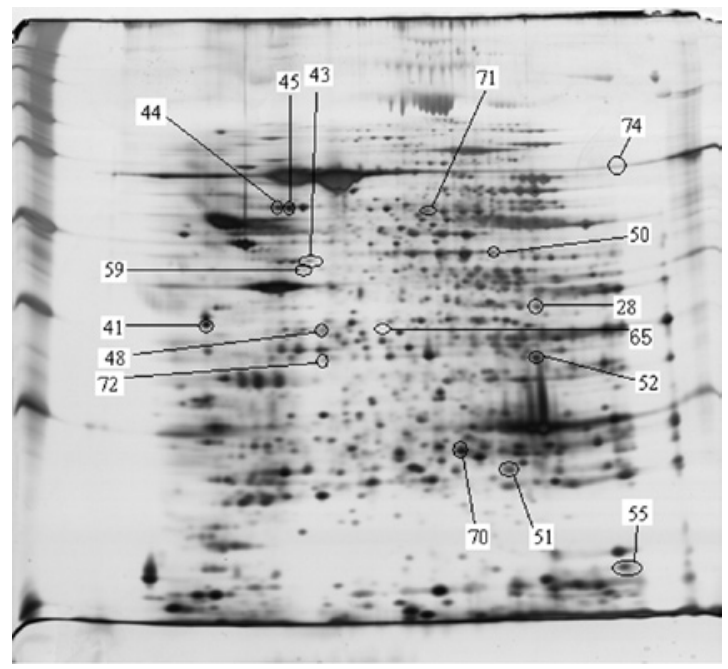

B

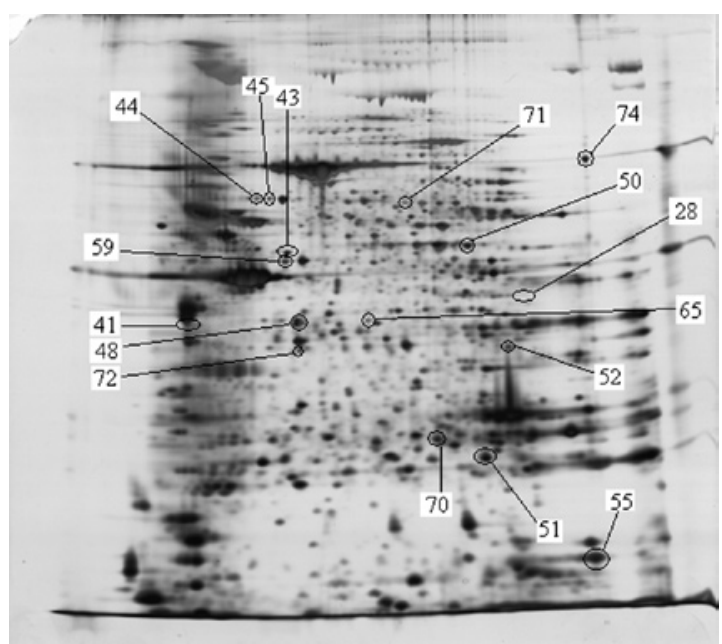

Figure 2. Images of 2DE in visceral adipose tissues in NOP (A) and NOR (B) mouse groups $(n=3)$. Differentially expressed proteins are circled. NOP, low-fat or normal-chow diet-fed obesity-prone mice; NOR, low-fat or normal-chow diet-fed obesity-resistant mice.

chondrial energy metabolism) and Enolase 3, $\beta$ muscle were significantly decreased by 8.1 - and 8.8 -fold, respectively, in the adipose tissues of the NOP mice compared to the NOR controls, while monoglyceride lipase (MGLL) (related to fat synthesis) and glucose-6-phosphate dehydrogenase (G6PDH) $\mathrm{X}$-linked were significantly decreased by 5.3 - and 4.7 -fold, respectively.

\section{Discussion}

High-fat diets play a key role in affecting body weight. However, some individuals are still rendered obese even when they ingest low-fat diets. The potential mechanisms remain unclear. In the present study, inbred C57BL/6 mice, also displayed a differential response to a low-fat diet as obesityprone and -resistant phenotypes, although these animals are usually used to induce diet-induced obesity (14) by a high-fat diet. After normalization for body weight, no significant difference in caloric intake was observed between the obesityprone and -resistant animals, suggesting that caloric intake may not be a key factor affecting visceral fat accumulation. 


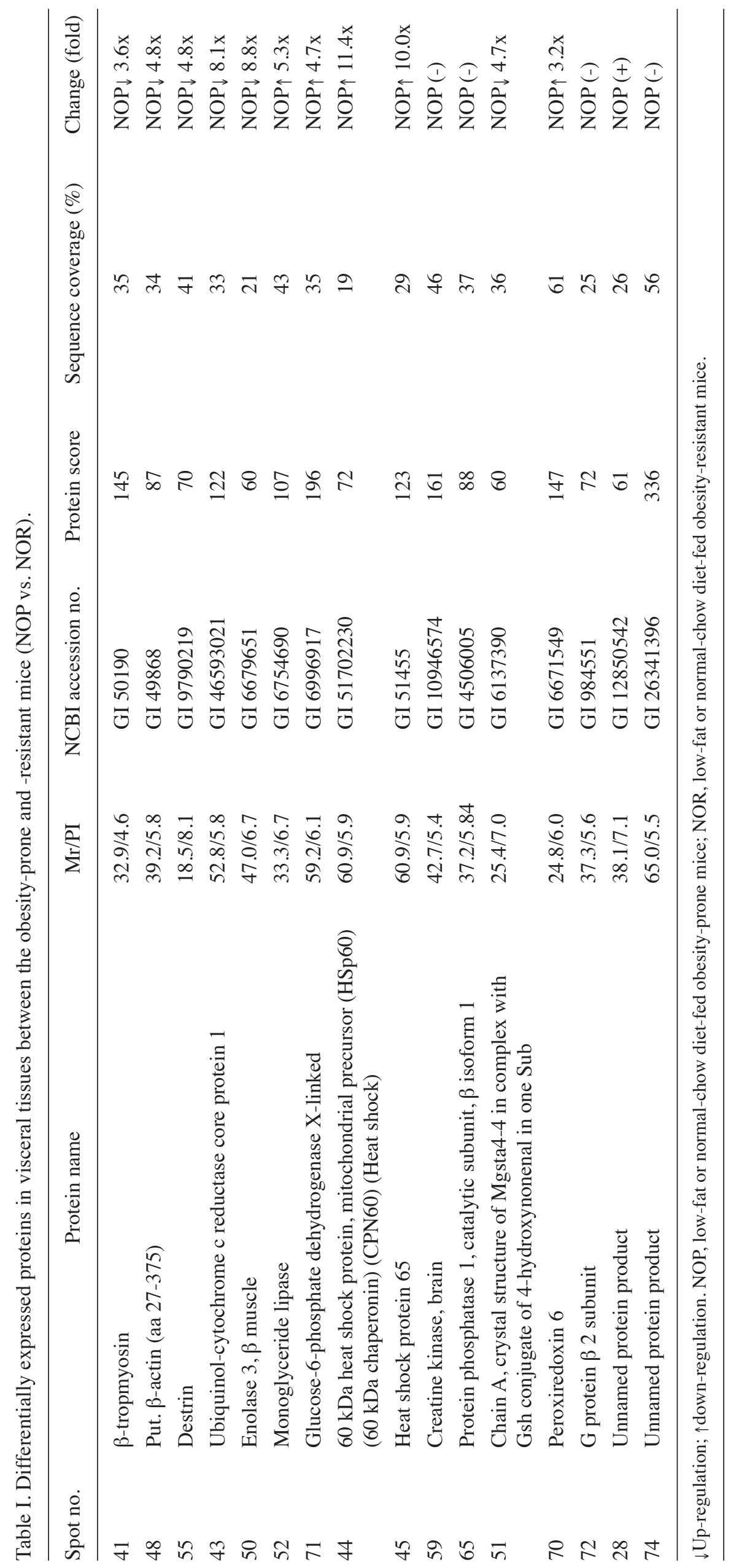



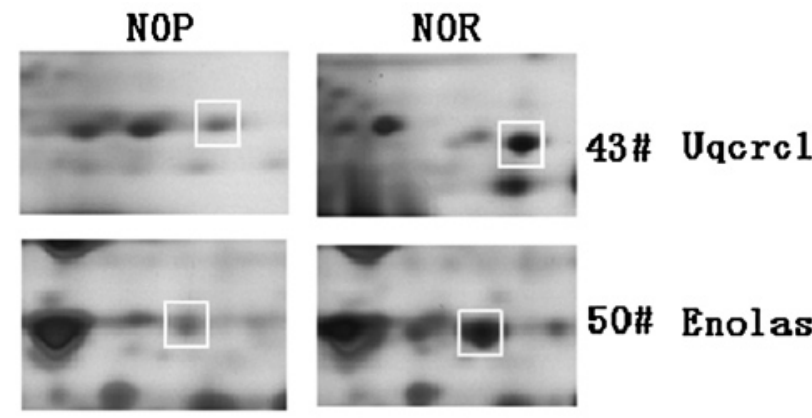

50\# Enolase 3
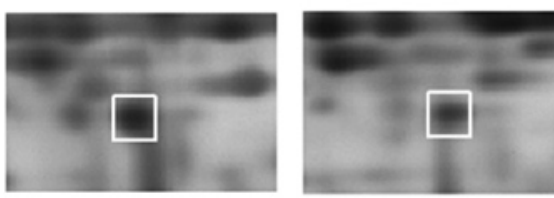

52\# MGLL
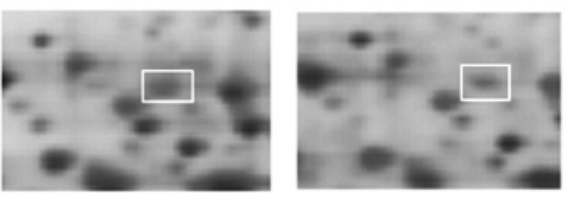

\section{1\# G6PDH}

Figure 3. Images of the differentially expressed proteins determined by $2 \mathrm{DE}$ in visceral adipose tissues of the normal-chow diet-fed obese-prone and -resistant mice after 6 weeks of treatment. Spots of interest are marked in a rectangle and enlarged $(n=3)$. NOP, low-fat or normal-chow diet-fed obesityprone mice; NOR, low-fat or normal-chow diet-fed obesity-resistant mice.

Also, the difference in intestinal lipid absorption rate between the obesity-prone and obesity-resistant mice was slight and did not contribute to visceral fat accumulation.

Therefore, we suspected that obesity-prone animals may have an altered energy metabolism and/or lipid synthesis in visceral adipose tissues in this model, since these tissues are an important marker for obesity. However, there are a multitude of factors involved in fat metabolism and/or synthesis, and the selection of specific factors for our research objectives was difficult. The proteomics method is very useful for supplying much information on a disease when the mechanisms involved are unknown.

To our knowledge, this study represents the first use of a proteomics method to investigate protein expression differences in visceral adipose tissues between obesity-resistant and obesity-prone animals fed low-fat diets. Uqcrc1, a subunit of the respiratory chain protein ubiquinol cytochrome c reductase, is responsible for mitochondrial energy metabolism and is associated with the development of obesity (15). Through a proteomics study, a significant decrease in Uqcrcl expression was found in the visceral adipose tissues of the NOP mice, which may be associated with attenuated mitochondrial energy metabolism. Enolase, also known as phosphopyruvate dehydratase, is a metalloenzyme responsible for the catalysis of the conversion of 2-phosphoglycerate to phosphoenolpyruvate, the ninth and penultimate step of glycolysis. Decreased expression of enolase indicates that glycolysis may be reduced in obesity-prone animals. MGLL functions together with hormone-sensitive lipase (HSL) to hydrolyze intracellular triglyceride stores in adipocytes and other cells to fatty acids and glycerol. MGLL may also complement lipoprotein lipase in completing hydrolysis of monoglycerides resulting from the degradation of lipoprotein triglycerides (16). Despite this, the obesity-prone mice showed a significant increase in visceral adipose tissues. Therefore, it may be speculated that i) MGLL may not play a key role in hydrolyzing triglycerides as HSL and adipose triglyceride lipase do; ii) MGLL may enhance the effects of lipoprotein lipase on fat storing in adipocytes. G6PDH is a cytosolic enzyme in the pentose phosphate pathway. Of greater quantitative importance is the production of NADPH for tissues actively engaged in biosynthesis of fatty acids and/or isoprenoids, such as the liver, mammary glands, adipose tissue and the adrenal glands. Therefore, the increased expression of G6PDH may promote lipid synthesis in visceral adipose tissues of obesity-prone animals.

Taken together, these results indicate that obesity-prone animals fed low-fat diets show differentially expressed proteins, e.g., Uqcrc1, Enolase 3, MGLL and G6PDH involved in energy metabolism, glycolysis and fat synthesis in visceral adipose tissues, which are useful for defining molecular markers and understanding the mechanisms of the development of obesity. Future studies should focus on how these proteins function in the visceral adipose tissues of obesityprone individuals.

\section{Acknowledgements}

This study was supported by the National Natural Science Foundation of China (30871428), the China Postdoctoral Science Foundation (20060390460), the Tertiary College Science Foundation of Nanshan, Shenzhen (2008028) and the Science and Research Seed Foundation (2008) of the Graduate School at Shenzhen, Tsinghua University.

\section{References}

1. Nair RP and Ren J: Pharmacotherapy of obesity - benefit, bias and hyperbole. Curr Med Chem16: 1888-1897, 2009.

2. Ginter E and Simko V: Adult obesity at the beginning of the $21 \mathrm{st}$ century: epidemiology, pathophysiology and health risk. Bratisl Lek Listy 109: 224-230, 2008.

3. Schrauwen P and Westerterp KR: The role of high-fat diets and physical activity in the regulation of body weight. Br J Nutr 84: 417-427, 2000.

4. Pagliassotti MJ, Gayles EC and Hill JO: Fat and energy balance. Ann NY Acad Sci 827: 431-448, 1997.

5. Ji H and Friedman MI: Reduced hepatocyte fatty acid oxidation in outbred rats prescreened for susceptibility to diet-induced obesity. Int J Obesity 32: 1331-1334, 2008.

6. Takahashi N, Patel HR, Qi Y, Dushay J and Ahima RS: Divergent effects of leptin in mice susceptible or resistant to obesity. Horm Metab Res 34: 691-697, 2002.

7. Primeaux SD, Barnes MJ and Bray GA: Olfactory bulbectomy increases food intake and hypothalamic neuropeptide $Y$ in obesity-prone but not obesity-resistant rats. Behav Brain Res 180: 190-196, 2007.

8. Trinder P: Determination of glucose in blood using glucose oxidase with an alternative oxygen acceptor. Ann Clin Biochem 6: 24-27, 1969.

9. Allain CC, Poon LC, Chan CS, Richmond W and Fu PC: Enzymatic determination of total cholesterol. Clin Chem 20: 470-475, 1974

10. Fossati P and Prencipe L: Serum triglycerides determined colorimetrically with an enzyme that produces hydrogen peroxide. Clin Chem 28: 2077-2080, 1982.

11. Izzo C, Grillo F and Murador E: Improved method for determination of high-density-lipoprotein cholesterol I. Isolation of high-density lipoproteins by use of polyethylene glycol 6000 . Clin Chem 27: 371-374, 1981.

12. Kerscher L, Schiefer S, Draeger B, Maier J and Ziegenhorn J: Precipitation methods for the determination of LDL-cholesterol. Clin Biochem 18: 118-125, 1985. 
13. Li G, Zhang XA, Wang H, et al: Comparative proteomic analysis of mesenchymal stem cells derived from human bone marrow, umbilical cord, and placenta: implication in the migration. Proteomics 9: 20-30, 2009.

14. Xie WD, Zhang YO, Wang NL, et al: Novel effects of macrostemonoside A, a compound from Allium macrostemon Bung, on hyperglycemia, hyperlipidemia and visceral obesity in high-fat diet-fed C57BL/6 mice. Eur J Pharmacol 599: 159-165, 2008.
15. Kunej T, Wang Z, Michal JJ, Daniels TF, Magnuson NS and Jiang Z: Functional UQCRC1 polymorphisms affect promoter activity and body lipid accumulation. Obesity 15: 2896-2901, 2007.

16. Karlsson M, Reue K, Xia YR, Lusis AJ, Langin D, Tornqvist H and Holm C: Exon-intron organization and chromosomal localization of the mouse monoglyceride lipase gene. Gene 272: $11-18,2001$ 